\title{
OBTENÇÃO DE MICROPARTÍCULAS ESTRUTURADAS CONTENDO CURCUMINA
}

\author{
J. T. do Prado Silva ${ }^{1}$, F. V. Leimann ${ }^{1}$, O. H. Gonçalves ${ }^{1}$ \\ ${ }^{1}$ Programa de Pós-graduação em Tecnologia de Alimentos (PPGTA) - Universidade \\ Tecnológica Federal do Paraná \\ jessicathais92@gmail.com
}

\begin{abstract}
RESUMO - A curcumina é um polifenol natural com importantes efeitos biológicos comprovados, atuando como um potente antioxidante, inibindo a formação de células cancerígenas, além de possuir efeito antiinflamatório. $O$ emprego da curcumina como corante em escala industrial é limitado devido à sua baixa solubilidade em água, sensibilidade ao tratamento térmico, luz, presença de íons metálicos, enzimas, ácido ascórbico, entre outros. Deste modo, a técnica de encapsulação vem sendo ressaltada na literatura como um método eficaz para melhorar sua aplicabilidade tecnológica. Neste trabalho, curcumina foi encapsulada em micropartículas de policaprolactona (PCL) recobertas com gelatina reticulada por transglutaminase através da técnica de emulsificação/evaporação do solvente. As micropartículas apresentaram formato esférico e eficiência de encapsulação de $(81 \pm 13) \%$. Além disso, foram avaliados os perfis de liberação da curcumina das micropartículas de PCL sem recobrimento e em micropartículas de PCL recobertas com gelatina reticulada com 1,96 e 5,9\% de transglutaminase (em relação à massa de gelatina). $\mathrm{O}$ recobrimento retardou a liberação da curcumina do interior das micropartículas.
\end{abstract}

\section{INTRODUÇÃO}

A curcumina apresenta inúmeras propriedades biológicas de interesse, com comprovada atividade antioxidante, anti-inflamatória, antitumoral e antimicrobiana. Apesar do grande interesse no uso da curcumina, sua aplicação industrial ainda é limitada devido à sua baixa solubilidade em água, e instabilidade frente a variações de $\mathrm{pH}$, temperatura e agentes oxidantes. Para viabilizar sua aplicação tecnológica, diferentes técnicas de micro e nanoencapsulação tem sido desenvolvidas (PARAMERA et al., 2011; SILVA-BUZANELLO et al., 2015). Em especial, a emulsificação-evaporação do solvente possui a vantagem de baixo consumo energético e possibilidade de uso de encapsulantes biocompatíveis e/ou biodegradáveis como os biopolímeros (YALLAPU et al., 2010). É importante nesse caso avaliar a forma como a curcumina encapsulada é liberada do interior das micropartículas (MASSIMINO e YOSHIOKA; 2014).

O objetivo deste trabalho foi encapsular curcumina em micropartículas de policaprolactona (PCL) recobertas com gelatina reticulada com transglutaminase. Foi avaliada a liberação da curcumina a fim de determinar o efeito do recobrimento nas taxas de liberação. 


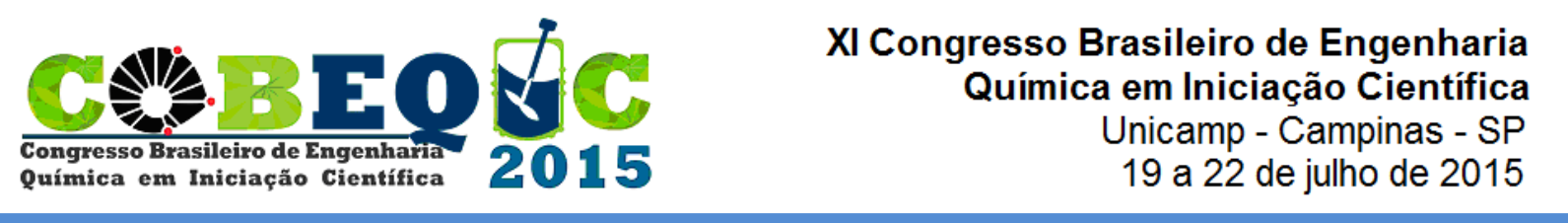

\section{MATERIAIS E MÉTODOS}

\subsection{Elaboração das micropartículas recobertas com gelatina reticulada contendo curcumina}

As micropartículas contendo curcumina foram elaboradas através da técnica de emulsificação/evaporação do solvente conforme descrito por Chen, Bei e Wang (2000). A Tabela 1 apresenta a formulação utilizada.

Tabela 1 - Formulação das micropartículas de curcumina.

\begin{tabular}{cc}
\hline Reagentes & Massa $(\mathbf{g})$ \\
\hline PCL & 1,5000 \\
\hline Diclorometano & 20,0000 \\
\hline Água destilada & 200,0000 \\
\hline Gelatina & 0,7500 \\
\hline Curcumina & 0,0150 \\
\hline
\end{tabular}

Para o recobrimento das micropartículas, utilizou-se o princípio da técnica de coacervação complexa (Kissel et al., 2006). A Tabela 2 apresenta a formulação utilizada para o recobrimento das micropartículas de curcumina.

Tabela 2 - Formulação do recobrimento das micropartículas de curcumina.

\begin{tabular}{cc}
\hline Reagentes & Massa $(\mathbf{g})$ \\
\hline Goma arábica & 0,7500 \\
\hline Água destilada & 30,0000 \\
\hline Transglutaminase & 0,0140 ou 0,0420 \\
\hline
\end{tabular}

\subsection{Caracterização das micropartículas}

O tamanho e morfologia das micropartículas foram analisadas em microscópio óptico com câmera acoplada ao computador. Para a determinação da concentração real de curcumina nas micropartículas, uma amostra homogênea de aproximadamente $0,5 \mathrm{~g}$ da solução de micropartículas foi liofilizada e armazenada sob refrigeração, sendo então dissolvida em $1 \mathrm{~mL}$ de diclorometano e adicionado $1 \mathrm{~mL}$ de metanol. A solução foi filtrada (Millipore, 0,45 $\mu \mathrm{m}$ ). A concentração total de curcumina (encapsulada e não encapsulada, [cur $]_{\text {total }}$ ) foi determinada por espectrofotometria em $465 \mathrm{~nm}$ de acordo com Silva-Buzanello et al. (2015). As análises foram realizadas em duplicata. Para a determinação da eficiência de encapsulação da curcumina, as micropartículas foram lavadas com etanol absoluto em filtro quantitativo (porosidade de $3 \mu \mathrm{m}$ ) e secas em estufa de circulação forçada a $60^{\circ} \mathrm{C}$ até massa constante. Dissolveu-se aproximadamente $10 \mathrm{mg}$ do material seco em $1 \mathrm{~mL}$ de diclorometano e adicionou-se $1 \mathrm{~mL}$ de metanol. A concentração de curcumina encapsulada ([cur] encapsulada) foi determinada como descrito acima. Esta análise foi realizada em duplicata e a eficiência de encapsulação $(\mathrm{EE}(\%))$ foi calculada pela Equação 1. 


$$
E E(\%)=\frac{[\text { cur }]_{\text {encapsulada }}}{[\text { cur }]_{\text {total }}} .100
$$

Para a caracterização das ligações químicas formadas após a reticulação da gelatina foram realizados ensaios de Espectroscopia no Infravermelho por Transformada de Fourier (FTIR) em pastilhas de KBr (IR AFFINITY-1, Shimadzu).

\subsection{Avaliação da liberação modificada de curcumina}

$16 \mathrm{~g}$ da dispersão de micropartículas foram transferidas para um Erlenmeyer de $250 \mathrm{~mL}$, que foi acondicionado em banho térmico com agitador orbital a $37^{\circ} \mathrm{C}$. Em seguida, adicionou-se ao Erlenmeyer o meio de liberação (solução aquosa de etanol $60 \% \mathrm{v} / \mathrm{v}$ ). Em tempos predeterminados, foram retiradas alíquotas de $2 \mathrm{~mL}$ da amostra. Cada retirada de amostra foi seguida pela reposição da mesma quantidade do meio de liberação, a fim de manter o volume total constante. As alíquotas de amostra foram imediatamente filtradas (Millipore, $0,45 \mu \mathrm{m}$ ) e analisadas por espectrofotometria UV-Vis.

\section{RESULTADOS E DISCUSSÃO}

\subsection{Obtenção das micropartículas e recobrimento}

As micropartículas de PCL apresentaram morfologia esférica, o que é esperado para a técnica de emulsificação/evaporação do solvente. Na Figura 1, apesar de a gelatina estar presente, esta não pode ser visualizada, pois a sua precipitação por coacervação ocorre em um estágio posterior. Após a reticulação utilizando 1,96\% de transglutaminase (Figura 2), é possível observar que a houve a inclusão das micropartículas de PCL em seu interior. O mesmo não ocorreu com 5,9\% de transglutaminase (Figura 3), onde houve a formação de partículas de gelatina dispersas na fase aquosa, não havendo incorporação das micropartículas de PCL. Isso pode ter ocorrido devido à maior velocidade da reação com o aumento da concentração de $\mathrm{TG}$, desfavorecendo a formação da camada de gelatina sobre as micropartículas de PCL.

Figura 1 - Micropartículas de PCL contendo curcumina $(100 x)$

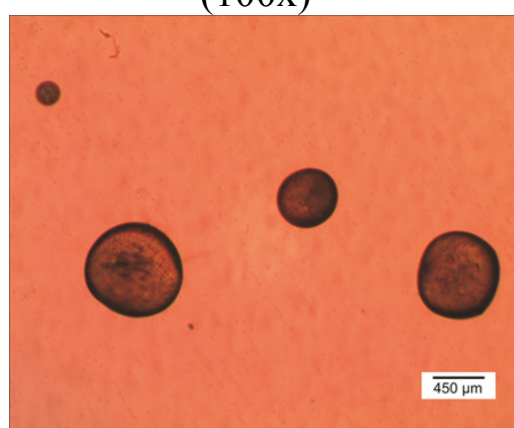

Figura 2 - Micropartículas de PCL contendo curcumina após o recobrimento com gelatina reticulada $(1,96 \%$ TG) $(100 x)$

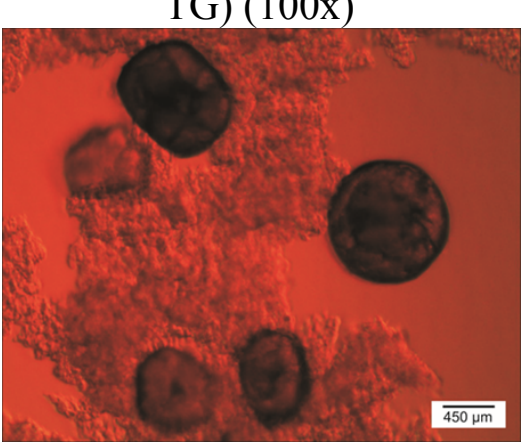

Figura 3 - Micropartículas de PCL contendo curcumina após o recobrimento com gelatina reticulada $(5,9 \%$

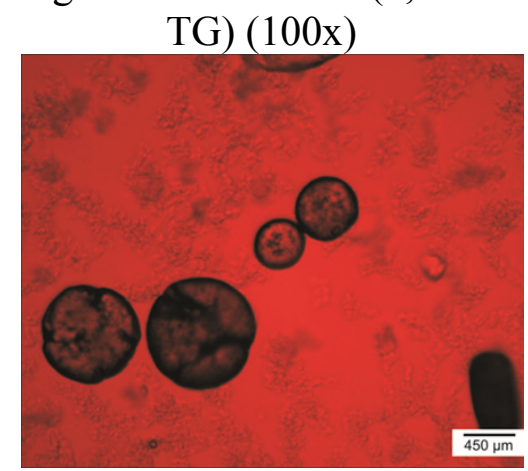

\subsection{Caracterização das micropartículas}


A partir do cálculo da concentração real de curcumina presente na dispersão de micropartículas, foi possível determinar, com auxílio da Equação 1, a eficiência de encapsulação, ou seja, a porcentagem de curcumina que foi incorporada nas micropartículas. As micropartículas confeccionadas neste estudo apresentaram média de $(81 \pm 13) \%$ de eficiência de encapsulação. Silva-Buzanello et al. (2015) produziram nanopartículas de poli (L-ácido láctico) variando a concentração de curcumina a ser encapsulada através da técnica de miniemulsificação/evaporação de solvente, alcançando eficiência de encapsulação de $67,0 \%$ a 98,3\%, dependendo da quantidade de curcumina adicionada. Outros autores procederam a encapsulação da curcumina onde se pode concluir que esta depende também do encapsulante e da técnica utilizada (DANDEKAR et al., 2010; YALLAPU et al., 2010), o que explica a diferença entre os valores encontrados nesse trabalho e os disponíveis em literatura.

A Figura 4 apresenta os espectros de FTIR para as micropartículas de PCL sem e com recobrimento de gelatina $(1,96 \% \mathrm{TG})$.

Figura 4 - Espectro de FTIR das micropartículas de PCL contendo curcumina antes e após o recobrimento com gelatina reticulada por transglutaminase

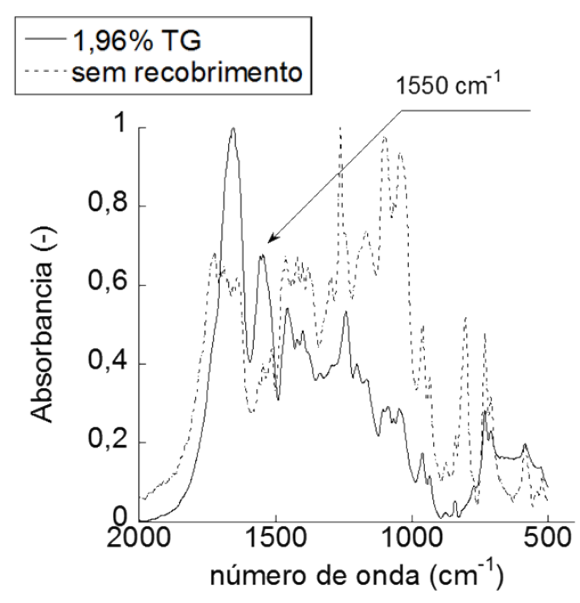

A banda de absorção localizada em $1550 \mathrm{~cm}^{-1}$, referente ao grupamento amida monosubstituída, pode ser encontrada nas partículas antes e depois da reticulação, já que faz parte da estrutura da gelatina. Após a reticulação, esta banda aumentou de intensidade devido à formação das ligações de reticulação promovidas pela tranglutaminase. Dessa forma é possível confirmar que a transglutaminase promoveu a reticulação da gelatina. A gelatina apresenta tanto resíduos de grupamento glutamina e quanto de lisina (FUCHSBAUER et. al., 1996; CARVALHO e GROSSO, 2006; CORTESI et. al., 1999). Dessa forma o produto da reação de reticulação é a formação de um grupamento amida monosubstituída bem como amônia. A transglutaminase é uma enzima capaz de catalisar reações de acil-transferência, introduzindo ligações cruzadas covalentes entre proteínas, peptídeos e aminas primárias (ZHU et al., 1995).

\subsection{Avaliação da liberação modificada da curcumina}

Na Figura 5, são exibidos os perfis de liberação da curcumina ao longo do tempo para as micropartículas de PCL sem recobrimento e as micropartículas de PCL recobertas com 
gelatina reticulada com 1,96 e 5,9\% (em relação à massa de gelatina). O período inicial da liberação é destacado na Figura 6, apresentando as retas de ajustes lineares para cada experimento.

Figura 5 - Perfis de liberação da curcumina em micropartículas de PCL antes e depois do

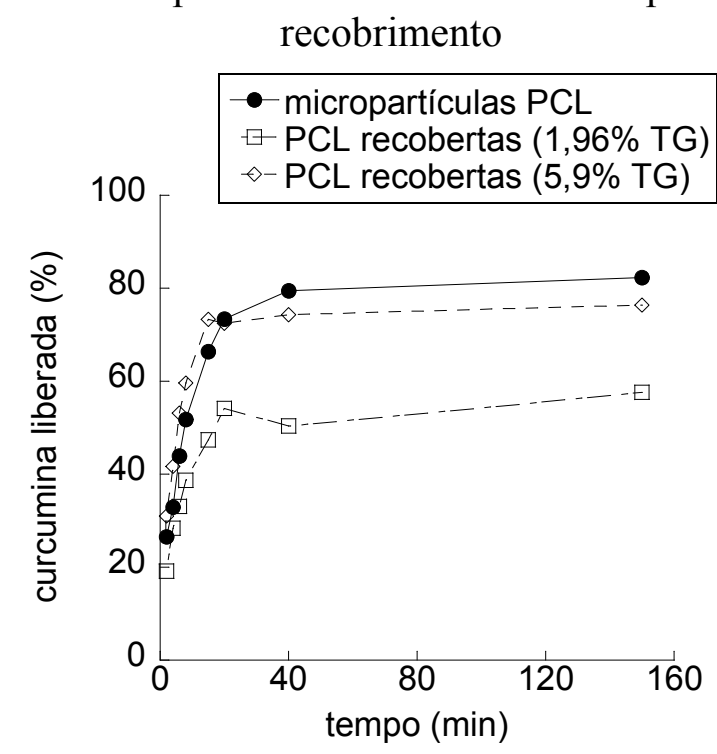

Figura 6 - Liberação inicial da curcumina em micropartículas de PCL antes e depois

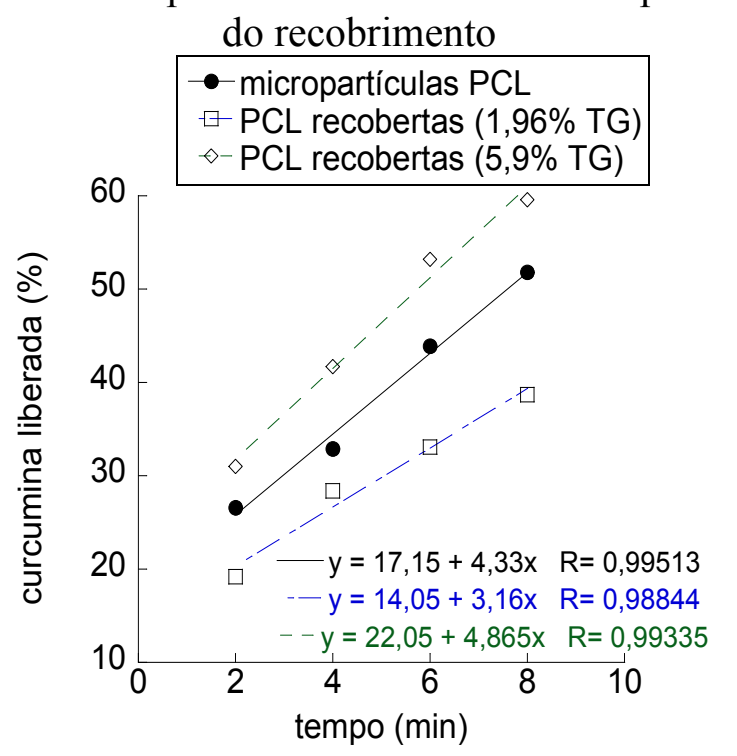

Para todos os casos, a liberação da curcumina ocorreu até aproximadamente 40 minutos, a partir do qual os percentuais liberados permaneceram constantes. Para as partículas sem recobrimento foi liberada cerca de $80 \%$ da curcumina total. No caso das partículas recobertas contendo $1,96 \%$ de transglutaminase (TG), o percentual máximo liberado foi em torno de $55 \%$ da curcumina total. Não foram perceptíveis variações significativas ao usar 5,9\% de TG. Esse resultado está de acordo com as imagens apresentadas pela Figura 6, onde é possível observar que não ocorreu o recobrimento das partículas ao usar 5,9\% de TG. Em um estudo conduzido por Parize (2009), a liberação controlada de curcumina em micropartículas de quitosana contendo reticulante iônico tripolifostato de sódio (TPF) em solução tampão pH 1,2 e 6,8 a $25^{\circ} \mathrm{C}$ foi analisada. Essa técnica melhorou o perfil de liberação da curcumina a partir de micropartículas de quitosana, onde quanto maior a concentração de TPF, menor a velocidade de liberação do corante. Alvim e Grosso (2010) produziram micropartículas por coacervação complexa reticuladas por glutaraldeído e transglutaminase, avaliando a liberação do recheio (oleoresina de páprica) em solução de álcool anidro. Neste caso, a reticulação por glutaraldeído também foi eficiente para a retenção do recheio das micropartículas.

\section{CONCLUSÃO}

A microencapsulação de curcumina em policaprolactona (PCL) através da técnica de emulsificação/evaporação do solvente resultou em partículas de formato esférico e a eficiência de encapsulação média de $(81 \pm 13) \%$. As imagens obtidas por microscopia óptica e os espectros de infravermelho permitiram constatar que houve a reticulação das micropartículas por gelatina. Os perfis de liberação da curcumina em micropartículas de PCL permitiram constatar que a reticulação influenciou na taxa de liberação do material de recheio das micropartículas. Entretanto, altas concentrações de transglutaminase (5,9\%) não 


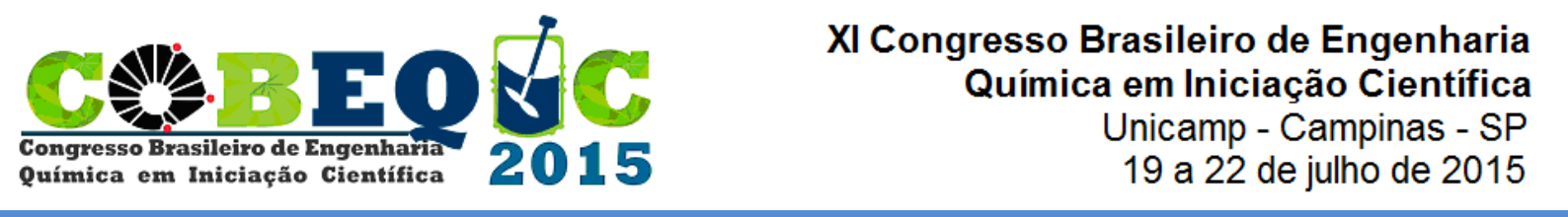

favoreceram o recobrimento das micropartículas e, consequentemente, não houve mudança no perfil de liberação quando comparado ao das partículas não recobertas. Os resultados permitiram concluir que a técnica de emulsificação/evaporação do solvente seguida do recobrimento por reticulação enzimática é uma boa alternativa para encapsular a curcumina e modificar seu perfil de liberação, informações importantes para a aplicação tecnológica do corante curcumina na indústria de alimentos.

\section{REFERÊNCIAS}

ALVIM, I. D.; GROSSO, C. R. F. Microparticles obtained by complex coacervation: influence of the type of reticulation and the drying process on the release of the core material. Ciênc. Tecnol. Aliment., v. 30, n. 4, p. 1069-1076, Out/Dez, 2010.

CARVALHO, R. A. de; GROSSO, C. R. F. Efeito do tratamento térmico e enzimático nas propriedades de filmes de gelatina. Sociedade Brasileira de Ciência e Tecnologia de Alimentos, v. 26, 2006.

CHEN, D.R.; BEI, J.Z.; WANG S.G. Polycaprolactone microparticles and their biodegradation. Polymer Degradation and Stability, v. 67, p. 455-459, 2000.

CORTESI, R. et al. Dextran cross-linked gelatin microspheres as a drug delivery system. European journal of pharmaceutics and biopharmaceutics, v. 47, n. 2, p. 153-160, 1999.

DANDEKAR, P. P. et al. Curcumin - loaded hydrogel nanoparticles: Application in anti malarial therapy and toxicological evaluation. Journal of Pharmaceutical Sciences, v. 99, p. $4992-5010.2010$.

FUCHSBAUER, H. L. et al. Influence of gelatin matrices cross-linked with transglutaminase on the properties of an enclosed bioactive material using P-galactosidase as model system. Biomaterials, p. 1481-1488, 1996.

KISSEL, T. et al. Microencapsulation thechniques for parenteral depot systems and their application in the pharmaceutical industry. Drugs and the Pharmaceutical Sciences Journal, n. 2, v. 158, p. 99-118. 2006.

MASSIMINO, L. C.; YOSHIOKA, S. A. Nanopartículas do antitumural curcumina: obtenção e caracterização. XXIV Congresso Brasileiro de Engenharia Biomédica CBEB, Uberlândia (MG), 2014.

PARAMERA, E. I.; KONTELES, S. J.; KARATHANOS, V. T. Stability and release properties of curcumin encapsulated in Saccharomyces cerevisiae, $\beta$-cyclodextrin and modified starch. Food Chemistry, v. 125, p. 913-922. 2011.

PARIZE, A. L. Desenvolvimento de sistemas microparticulados e de filmes a base de quitosana e corante natural cúrcuma. 2009. 187 f. Tese (Doutorado em Química). Universidade Federal de Santa Catarina, Florianópolis (SC), 2009.

SILVA - BUZANELLO, R. A. et al. Validation of an Ultraviolet - visible (UV - Vis) technique for the quantitative determination of curcumin in poly ( $\mathrm{L}$ - lactic acid) nanoparticles. Food Chemistry, v. 172, p. 99 - 104. 2015. 
ZUH, Y. et al. Microbial transglutaminase - a review of its production and application in food processing. Appl Microbiol. Biotechnol., v. 44, p. 277-282, Mar. 1995.

YALLAPU, M. M. et al. Fabrication of curcumin encapsulated PLGA nanoparticles for improved therapeutic effects in metastatic cancer cells. Journal of Colloid Interface Science, v. 351, p. $19-29.2010$. 